\title{
Identification of Phylogenetic Position in the Chlamydiaceae Family for Chlamydia Strains Released from Monkeys and Humans with Chlamydial Pathology
}

\author{
Alexander Karaulov, ${ }^{1}$ Vladimir Aleshkin, ${ }^{2}$ Vladimir Slobodenyuk, ${ }^{2}$ Olga Grechishnikova, ${ }^{2}$ \\ Stanislav Afanasyev, ${ }^{2}$ Boris Lapin, ${ }^{3}$ Eteri Dzhikidze, ${ }^{3}$ Yuriy Nesvizhsky, ${ }^{1}$ Irina Evsegneeva, ${ }^{1}$ \\ Elena Voropayeva, ${ }^{2}$ Maxim Afanasyev, ${ }^{2}$ Andrei Aleshkin, ${ }^{2}$ Valeria Metelskaya, ${ }^{2}$ \\ Ekaterina Yegorova, ${ }^{2}$ and Alexandra Bayrakova ${ }^{2}$
}

${ }^{1}$ Sechenov Moscow Medical Academy, Trubetskaya 8, 119991 Moscow, Russia

${ }^{2}$ Garbichevsky Moscow G.N. Research Institute of Epidemiology and Micro-Biology, Admiral Makarov 10, 125212 Moscow, Russia

${ }^{3}$ Research Institute of Medical Primatology, Veseloye-1, 354376 Sochi-Adler, Russia

Correspondence should be addressed to Alexander Karaulov, karaulov@mtu-net.ru

Received 27 January 2010; Revised 27 April 2010; Accepted 5 May 2010

Academic Editor: Catalin S. Buhimschi

Copyright () 2010 Alexander Karaulov et al. This is an open access article distributed under the Creative Commons Attribution License, which permits unrestricted use, distribution, and reproduction in any medium, provided the original work is properly cited.

Based on the results of the comparative analysis concerning relatedness and evolutional difference of the 16S-23S nucleotide sequences of the middle ribosomal cluster and 23S rRNA I domain, and based on identification of phylogenetic position for Chlamydophila pneumoniae and Chlamydia trichomatis strains released from monkeys, relatedness of the above stated isolates with similar strains released from humans and with strains having nucleotide sequences presented in the GenBank electronic database has been detected for the first time ever. Position of these isolates in the Chlamydiaceae family phylogenetic tree has been identified. The evolutional position of the investigated original Chlamydia and Chlamydophila strains close to analogous strains from the Gen-Bank electronic database has been demonstrated. Differences in the 16S-23S nucleotide sequence of the middle ribosomal cluster and $23 \mathrm{~S}$ rRNA I domain of plasmid and nonplasmid Chlamydia trachomatis strains released from humans and monkeys relative to different genotype groups (group B-B, Ba, D, Da, E, L1, L2, L2a; intermediate group-F, G, Ga) have been revealed for the first time ever. Abnormality in incA chromosomal gene expression resulting in Chlamydia life development cycle disorder, and decrease of Chlamydia virulence can be related to probable changes in the nucleotide sequence of the gene under consideration

\section{Introduction}

Chlamydia is obligate intracellular bacteria that replicate only in the cytoplasmic inclusions of the eukaryotic host cells. They are grouped in the Chlamydiales order, Chlamydieceae family; they comprise two kinds, Chlamydia and Chlamydophila, which in turn consist of nine species subdivided into groups depending on whether they cause pathological conditions in human beings. The group of pathogens that infect humans includes the most commonly diagnosed species Chlamydia trachomatis and the least frequent Chlamydophila pneumoniae (formerly Chlamydia pheumoniae); the very rare species Chlamydophila psittaci (formerly Chlamydia psittaci) causes disease in birds. Chlamydophila abortus (formerly Chlamydia abortus) causes miscarriages while Chlamydophila felis causes pneumonia in cats. The species that have never been found to cause medical conditions in humans include: Chlamydophila caviae (formerly Chlamydia caviae) that causes conjunctivitis in guinea pigs, Chlamydophila pecorum (formerly Chlamydia pecorum), Chlamydia suis (formerly Chlamydia trachomatis) that causes disease in pigs, Chlamydophila 
pecorum (formerly Chlamydia pecorum), and Chlamydia suis (formerly Chlamydia trachomatis), which causes disease in mice [1-3].

In the past the following criteria were used to differentiate between species: the morphology of inclusions, sensitivity to sulfadiazine, ability to synthesise and accumulate glycogen in chlamydial inclusions, and estimates of the DNA-DNA homology. Thus, Chlamydia trachomatis that belongs to the Chlamydia genus and Chlamydophila psittaci that belongs to the Chlamydophila genus were the first two species to be differentiated on the basis of differences in the morphology of inclusions, glycogen synthesis, and sensitivity to sulfadiazine. All chlamydia-like bacteria were classified as either Chlamydia trachomatis or Chlamydia psittaci (Chlamydophila psittaci) of the Chlamydiales order depending on their morphology and development cycle. Strains of Chlamydia trachomatis were identified on the basis of their ability to accumulate glycogen in inclusions and their sensitivity to sulfadiazine; strains of Chlamydia psittaci were those that lacked the ability to accumulate glycogen and could not resist sulfadiazine $[1,4]$. The Chlamydophila pneumoniae species was identified as Chlamydophila psittaci because it had similar phenotypic characteristics such as the density of inclusions, inability to synthesise glycogen, and resistance to sulfadiazine. However, later on Chlamydophila pneumoniae was recognised as a separate species as it exhibited ultrastructural differences in the morphology of elementary bodies and DNA-DNA homology compared to other chlamydia [5]. The fourth species to be recognised as a species different from Chlamydophila psittaci (Chlamydia psittaci) in its phenotypic characteristics was Chlamydophila pecorum [2].

The modern classification of bacterial species has been revised and at the moment it is based on genetic methods of estimating the DNA-DNA and rRNA-DNA homologies and comparing the results of sequencing the $16 \mathrm{~S}$ and $23 \mathrm{~S}$ sections of rRNA, using the multipoint mapping sequencing method $[1,3,5-13]$.

Of all the molecular analysis methods available, comparative sequencing of rRNA or ribosomal DNA is most suitable for studying the phylogeny of chlamydia, which are microorganisms with similar phenotypes. When studying the phylogeny of chlamydia we primarily analyse the sequences 16S-23S in rRNA and 23S rRNA genes. All species of chlamydia are classified as members of the Chlamydiaceae family if the homology of the 16S rRNA gene is more than $90 \%[12,14]$. Other groups of chlamydia-like organisms exhibit a homology of the 16S rRNA gene with chlamydia of more than $80 \%$. These include strain Simkania Z [8], strain Hall's coccus [7], and strain Candidatus, that is a close relative of Parachlamydia acanthamoebae [6], which were all obtained from amoebas and were previously interpreted as rickettsia. These bacteria were classified as members of the Chlamydiales order because they are obligate intracellular microorganisms with a development cycle similar to that of chlamydia. Studying the homology of the $16 \mathrm{~S}$ rRNA gene in chlamydia-like organisms may result in the discovery of new groups of organisms, or new strains of chlamydia, which will be classified as Chlamydiales. There are many very known strains of Chlamydophila psittaci, Chlamydia trachomatis, and Chlamydophila pneumoniae obtained from birds, animals, and humans, the sequencing of whose rRNA is described in sundry papers and books $[1,4,12,15]$. However, nobody has ever had sequences and carried out a phylogenetic analysis of the strains Chlamydia trachomatis and Chlamydophila pneumoniae obtained from monkeys, which would have determined if they were related to the human strains of chlamydia.

The purpose of this study is to carry out a phylogenetic analysis of the 16S-23S midribosome section and the I $23 \mathrm{~S}$ domain of rRNA in the strains of chlamydia obtained from monkeys and humans suffering from pathologies caused by chlamydia.

\section{Materials and Methods}

We studied two strains of chlamydia obtained from the cervical canal of monkeys. One of the strains did not have plasmids (Chlamydia trachomatis-NPL) and the other had them (Chlamydia trachomatis-PL); this latter strain was obtained from the lungs of a monkey that died from pneumonia (Chlamydophila pneumoniae-PN); the plasmid carrying strain Chlamydia trachomatis-PL2 obtained from the cervical canal of a human being and the strain Chlamydophila pneumoniae-PN2 obtained from the mouth of a human being $[16,17]$. The reference strains Chlamydophila pneumoniae-B and Chlamydia trachomatisBurkhan (Chlamydia trachomatis-PL3) were provided by the State Collection of Chlamydia of the D.I. Ivanovskiy State Virology Research Institute of the Russian Academy of Medical Sciences.

The midribosome section of the 16S-23S rRNA genes (include domain I) was amplified and sequenced using purpose-built oligonulceotides: forward primer: 16SF2 5'-CCG CCC GTC ACA TCA TGG-3', forward primer: IGSIGF $5^{\prime}$-ATA ATA ATA GAC GTT TAA GA-3', and reverse primer: 23R 5'-TAC TAA GAT GTT TCA GTT C$3^{\prime}[4]$.

Amplification was carried out in a $40 \mathrm{mcl}$ mixture comprising: PCR Buffer $(\times 10): 700 \mathrm{mM}$ Tris- $\mathrm{HCl}, \mathrm{pH} 8.6 / 25^{\circ} \mathrm{C}$, $166 \mathrm{mM}\left(\mathrm{NH}_{4}\right)_{2} \mathrm{SO}_{4}, 25 \mathrm{mM} \mathrm{MgCl}, 0.2 \mathrm{mM}$ dNTPs, and $2.5 \mathrm{U}$ Taq-polymerase, using a GeneAmp 2700 amplifier (Applied Biosystems, US). The size of the products of amplification with primers 16 SF2 and 23R, IGSIGF and 23R was 602 and 276 pairs of nucleotides, respectively.

The section with the primers 16 SF2 and 23R was amplified under the following conditions: $95^{\circ} \mathrm{C}$ for 3 minutes, then 40 cycles: $94^{\circ} \mathrm{C}$ for 20 seconds, $55^{\circ} \mathrm{Cfor} 20$ seconds., $72^{\circ} \mathrm{Cfor}$ 20 seconds, and $72^{\circ} \mathrm{Cfor} 3$ minutes and finally cooling to $6^{\circ} \mathrm{C}$ with subsequent storage at $10^{\circ} \mathrm{C}$. v IGSIGF and $23 \mathrm{R}$ was amplified under the following conditions: $95^{\circ} \mathrm{Cfor} 3$ minutes, then 40 cycles: $94^{\circ} \mathrm{Cfor} 15$ seconds, $50^{\circ} \mathrm{Cfor} 15$ seconds, $72^{\circ} \mathrm{Cfor} 15$ seconds, and finally $72^{\circ} \mathrm{Cfor} 2$ minutes followed by cooling to $6^{\circ} \mathrm{C}$ with subsequent storage at $10^{\circ} \mathrm{C}$.

Following the amplification $6 \mathrm{mcl}$ of the sample was mixed with a 6-unit buffer for loading and then loaded into a $1.5 \%$ agarous gel $(0.5 \mathrm{mkg} / \mathrm{ml} \mathrm{EBR})$. Electrophoresis was conducted at $50 \mathrm{~A}, 100 \mathrm{~V}$ per $1 \mathrm{~cm}^{2}$ for 30 minutes. 
The amplification products were visualised on an ECX-20L transilluminator (Vilber Lourmat, Germany), using a Gel Imager electrophoresis result registration system (Helicon, Russia).

The nucleotide sequence of the fragments was determined using an ABI Prism 3100 Genetic Analyzer (Applied Biosystems, US) and a BigDye Terminator v3.1 Cycle Sequencing Kit set of sequencing reagents (Applied Biosystems, US), as per the manufacturer's instructions based on PYNNY CJSC (Post genome and nanotechnology innovations, based on the Innovation Centre for Medical Nanobiotechnologies of the State Research Institute for Physical and Chemical Medicine of the Ministry of Health of the Russian Federation, Moscow).

Sequencing was done using both forward primers (16SF2 5'-CCG CCC GTC ACA TCA TGG-3', IGSIGF 5'-ATAATA ATA GAC GTT TAA GA-3'), and reverse primers (23R $5^{\prime}$-TAC TAA GAT GTT TCA GTT C-3'), to get more specific results. The results of the sequencing of fragments were analysed and compared with reverse primer sequencing using the Vector NTI Advance 9.0 (PC) software package (http://www.invitrogen.com/site/us/en/home.html).

The nucleotide sequences 16S-23S of the rRNA genes obtained in the experiment were analysed using MEGA (Molecular Evolutionary Genetics Analysis) version 4.1 (http://www.megasoftware.net/) [18]. Multiple straightening of the nucleotide sequences of the analysed strains and isolated sections of RNA was done with other nucleotide sequences of the RNA of different species of chlamydia available in the GenBank NCBI database. Genetic relation was analysed and phylogenetic trees were constructed using the MEGA 4.1 programme employing the Neighbour-Joining method, based on the $p$-distance model doing a Bootstrap Test of Phylogeny (1000 repetitions) and the Maximum Parsimony method. The degree of homology in the 16S-23S sequences of the midribosome section and domain I of the 23S rRNA gene of the studied strains with different species of chlamydia published in GenBank NCBI were analysed using BLAST (http://blast.ncbi.nlm.nih.gov/Blast.cgi/).

Phylogenetic analysis of the 16S-23S midribosome section and domain I of the 23S rRNA gene was carried out and tree diagrams were built in comparison with representatives of the Chlamydiales order found in GenBank NCBI (http://www.ncbi.nlm.nih.gov/Genbank/): Chlamydophila abortus EBA (U76710), Chlamydophila psittaci 6BC (U68447), Chlamydophila psittaci NJ1 (U68419), Chlamydophila caviae GPIC (D85708), Chlamydophila felis FP Baker (U68457), Chlamydophila pneumoniae N16 (U68426), Chlamydophila pneumoniae TW-183 (U76711), Chlamydophila pecorum E58 (U68433), Chlamydophila pecorum IPA (U68434), Chlamydia trachomatis A/Har13 (U68438), Chlamydia trachomatis B/TW-5/OT (68440), Chlamydia trachomatis D/UW-3/CX (U68441), Chlamydia trachomatis L2/434/BU (U68443), Chlamydia suis R22 (U68420), Chlamydia suis S45 (U73110), Chlamydia muridarum MoPn (U68436), and Chlamydia muridarum SFPD (U68437). Of these chlamydia strains the following are well-documented plasmid carriers: Chlamydophila psittaci 6BC-pCpA1 [19, 20], Chlamydophila pneumoniae
N16-pCpnE1 [19, 20], Chlamydia trachomatis B/TW5/OT-pCTT1 [21], Chlamydia trachomatis D/UW-3/CXpCHL1 [22], Chlamydophila felis-pCfe1 [23], Chlamydophila caviae GPIC-pCpGP1 [24], Chlamydia trachomatis A/Har-13-pCTA [25], Chlamydia trachomatis L2/434/BUpL2 [26], Chlamydia muridarum MoPn-pMoPn [27]. Information about sequenced plasmids is also found in GenBank NCBI. MEGA 4.1 software packaged was used to estimate the evolutionary difference between the sequences and the standard estimation error(s) for the c I of chlamydia. Variation statistics methods based on calculating the arithmetic mean and standard error $(M \pm m)$ [28] were used to mathematically process the difference in the homology of the studied strains Chlamydia trachomatis (NPL, PL, PL2, and PL3) and Chlamydophila pneumoniae (PN, PN2, and B) with the strains of chlamydia found in GenBank NCBI. All mathematical processing was done in Microsoft Excel 2007.

\section{Results and Discussion}

Our previous studies established that the strain Chlamydia trachomatis-NPL obtained from monkeys does not have any plasmids. This strain is less virulent than the plasmidcarrying strains Chlamydia trachomatis-PL and Chlamydia trachomatis-PL2, isolated from humans and the reference strain Chlamydia trachomatis-Burkhan (Chlamydia trachomatis-PL3) [16, 17]. Plasmids contain a gene that codes for the membrane protein pgp 3 in chlamydia; this protein plays a role in the immune response of the organism to pathogens by inducing the generation of inflammatory cytokines in the macrophages and activating an inflammatory reaction [29]. Plasmids also play a dominant role in the accumulation of glycogen in chlamydia inclusions, which is explained by the presence in plasmids of sequences specific chromosome genes (pgi, mrsA1, glgA, glgB, glgX, and $\operatorname{glgP}$ ) responsible for the metabolism of glycogen and performing the function of their transcription regulator. The Chlamydia trachomatis strains that do not carry the critical plasmid cause asymptomatic development of the disease (one was obtained from a patient suffering from proctocolitis and characterised as an L2 serotype L2 [30], another was taken from a patient suffering from asymptomatic urethritis and characterised as serovar B [31], a third clinical isolate, C599 was taken from the urethra of a patient with an asymptomatic condition and after being sequenced was characterised as serovar E/Bour [32]). 50\% of the time an infecting dosage of a strain not carrying a plasmid is 400 times the dose of a plasmid-carrying strain [33]. While the majority of the Chlamydia trachomatis strains obtained from humans carry plasmids, $54 \%$ of the strains obtained from monkeys do not have plasmids. This explains the fact that monkeys suffering from chlamydia-caused conditions present with less clear symptoms than humans [17].

The cultivation of Chlamydia trachomatis strains in cells is accompanied either by the formation of multiple intracellular chlamydia inclusions or by the emergence of one big inclusion. This is first of all related to the expression of the incA gene at the level of the IncA protein (family of Inc-proteins-IncA, IncB, IncC, IncE, and IncG), which is 


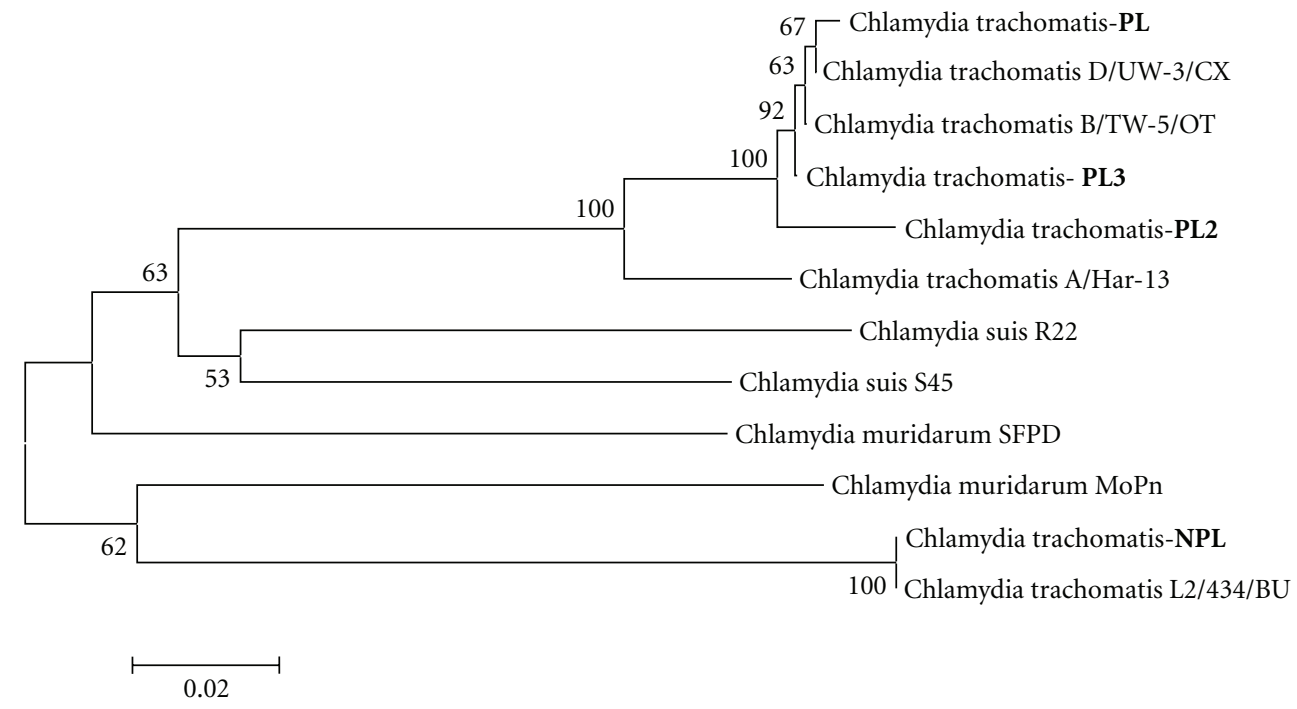

FIGURE 1: Dendrogram resulted from phylogenetic analysis of middle fragment of 16S-23S rRNA genes and 23S rRNA area of Chlamydia strains I segment by means of Neighbour-Joining method with execution of Bootstrap Test of Phylogeny (1000 repetitions), p-distance model demonstrating position of Chlamydia trachomatis strains being under investigation (PL: plasmid strain released from monkeys, PL2: plasmid strain released from humans, and PL3: reference strain and NPL: nonplasmid strain released from monkeys).

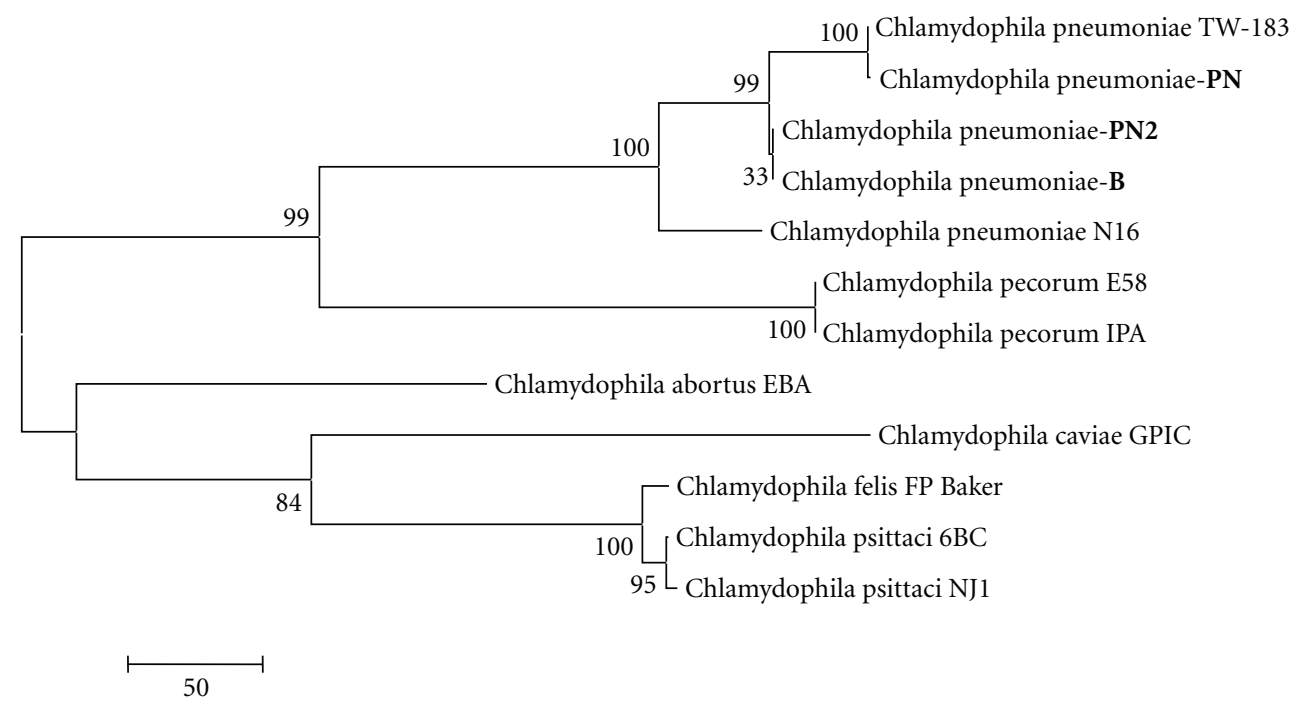

FIGURE 2: Dendrogram resulted from phylogenetic analysis of middle fragment of 16S-23S rRNA genes and 23S rRNA area of Chlamydophila Chlamydia strains I segment by means of Maximum Parsimony method with execution of Bootstrap Test of Phylogeny (1000 repetitions), p-distance model reflecting position of Chlamydophila pneumoniae strains being under investigation (PN: monkey strain, PN2: human strain, and B: reference strain).

part of the composition of the membrane inclusions and is responsible for vacuolisation. If there is no expression of this gene at all, the chlamydia inclusions multiply [34, 35]. The IncA protein has a function in the formation of secondary inclusions that allow chlamydia to form intracellular recesses where they can grow and continuously infect the cells forming after the division of the mother cell [36]. When the plasmid-free strain Chlamydia trachomatisNPL-genotype E cultivates multiple intracellular chlamydia inclusions (vacuoles) are observed inside the cell while in the case of the plasmid-carrying strains Chlamydia trachomatisPL (obtained from monkeys) and Chlamydia trachomatisPL2 (obtained from humans), which are G genotype, one big intracellular chlamydia inclusion forms [16].

Genotyping the Chlamydia trachomatis strains established that the strains obtained from monkeys were primarily genotypes E (42.3\%) and G (57.7\%); the plasmid carrying strain Chlamydia trachomatis-PL has genotype G; 
TABLE 1: Comparative analysis results concerning homology of the nucleotide sequence $16 \mathrm{~S}-23 \mathrm{~S}$ of the middle ribosomal cluster and $23 \mathrm{~S}$ rRNA I gene domain of the Chlamydia strains being investigated with analogous sequences of other strains.

\begin{tabular}{|c|c|c|c|c|c|c|c|c|c|c|c|c|c|c|}
\hline \multirow{3}{*}{$\begin{array}{l}\text { Chlamydia strains } \\
\text { from the GenBank } \\
\text { database }\end{array}$} & \multicolumn{14}{|c|}{$\begin{array}{l}\text { Homology percentage of the strains being investigated related to known Chlamydia strains from the GenBank } \\
\text { database }\end{array}$} \\
\hline & \multicolumn{2}{|c|}{$\begin{array}{l}\text { Chlamydia } \\
\text { trachomatis- } \\
\text { NPL }\end{array}$} & \multicolumn{2}{|c|}{$\begin{array}{l}\text { Chlamydia } \\
\text { trachomatis- } \\
\text { PL }\end{array}$} & \multicolumn{2}{|c|}{$\begin{array}{l}\text { Chlamydophila } \\
\text { pneumoniae } \\
\text { PN }\end{array}$} & \multicolumn{2}{|c|}{$\begin{array}{l}\text { Chlamydia } \\
\text { trachomatis- } \\
\text { PL2 }\end{array}$} & \multicolumn{2}{|c|}{$\begin{array}{l}\text { Chlamydophila } \\
\text { pneumoniae- } \\
\text { PN2 }\end{array}$} & \multicolumn{2}{|c|}{$\begin{array}{l}\text { Chlamydophila } \\
\text { pneumoniae-B }\end{array}$} & \multicolumn{2}{|c|}{$\begin{array}{l}\text { Chlamydia } \\
\text { trachomatis- } \\
\text { PL3 }\end{array}$} \\
\hline & $\mathrm{hm} \hat{~}$ & $\mathrm{~d}^{* *}$ & hm & $\mathrm{d}^{* *}$ & $\mathrm{hm}$ & $\mathrm{d}^{* *}$ & $\mathrm{hm}$ & $\mathrm{d}^{* *}$ & hm & $\mathrm{d}^{* *}$ & hm & $\mathrm{d}^{* *}$ & hm & $\mathrm{d}^{* *}$ \\
\hline Chl. abortus EBA & 78 & 22 & 83 & 17 & 90 & 10 & 83 & 17 & 95 & 5 & 90 & 10 & 83 & 17 \\
\hline Chl. psittaci 6BC & 78 & 22 & 84 & 16 & 90 & 10 & 84 & 16 & 94 & 6 & 90 & 10 & 84 & 16 \\
\hline Chl. psittaci NJ1 & 78 & 22 & 84 & 16 & 91 & 9 & 83 & 17 & 96 & 4 & 91 & 9 & 84 & 16 \\
\hline Chl. caviae GPIC & 79 & 21 & 84 & 16 & 90 & 10 & 84 & 16 & 95 & 5 & 90 & 10 & 85 & 15 \\
\hline Chl. felis FP Baker & 79 & 21 & 85 & 15 & 90 & 10 & 85 & 15 & 85 & 15 & 90 & 10 & 85 & 15 \\
\hline $\begin{array}{l}\text { Chl. pneumoniae } \\
\text { N16 }\end{array}$ & 82 & 18 & 79 & 21 & 98 & 2 & 82 & 18 & 98 & 2 & 98 & 2 & 82 & 18 \\
\hline $\begin{array}{l}\text { Chl. pneumoniae } \\
\text { TW-183 }\end{array}$ & 83 & 17 & 80 & 20 & 99 & 1 & 84 & 16 & 99 & 1 & 99 & 1 & 82 & 18 \\
\hline Chl. pecorum E58 & 80 & 20 & 82 & 18 & 90 & 10 & 82 & 18 & 97 & 3 & 90 & 10 & 82 & 18 \\
\hline Chl. pecorum IPA & 80 & 20 & 82 & 18 & 90 & 10 & 82 & 18 & 97 & 3 & 90 & 10 & 82 & 18 \\
\hline $\begin{array}{l}\text { Ch. trachomatis } \\
\text { A/Har13 }\end{array}$ & 99 & 1 & 99 & 1 & 82 & 18 & 99 & 1 & 94 & 6 & 82 & 18 & 100 & 0 \\
\hline $\begin{array}{l}\text { Ch. trachomatis } \\
\text { B/TW-5/OT }\end{array}$ & 99 & 1 & 99 & 1 & 82 & 18 & 99 & 1 & 94 & 6 & 82 & 18 & 99 & 1 \\
\hline $\begin{array}{l}\text { Ch. trachomatis } \\
\text { D/UW-3/CX }\end{array}$ & 99 & 1 & 99 & 1 & 83 & 17 & 99 & 1 & 94 & 6 & 83 & 17 & 99 & 1 \\
\hline $\begin{array}{l}\text { Ch. trachomatis } \\
\mathrm{L} 2 / 434 / \mathrm{BU}\end{array}$ & 100 & 0 & 98 & 2 & 83 & 17 & 98 & 2 & 94 & 6 & 83 & 17 & 98 & 2 \\
\hline Ch. suis R22 & 94 & 6 & 95 & 5 & 84 & 16 & 95 & 5 & 94 & 6 & 84 & 16 & 95 & 5 \\
\hline Ch.suis S45 & 94 & 6 & 95 & 5 & 83 & 17 & 95 & 5 & 93 & 7 & 84 & 16 & 95 & 5 \\
\hline $\begin{array}{l}\text { Chl. muridarum } \\
\text { MoPn }\end{array}$ & 94 & 6 & 94 & 6 & 83 & 17 & 94 & 6 & 94 & 6 & 83 & 17 & 95 & 5 \\
\hline $\begin{array}{l}\text { Chl. muridarum } \\
\text { SFPD }\end{array}$ & 94 & 6 & 94 & 6 & 83 & 17 & 94 & 6 & 94 & 6 & 83 & 17 & 95 & 5 \\
\hline $\begin{array}{l}\text { difference in } \\
\text { homology }(M \\
\pm m)^{*}\end{array}$ & \multicolumn{2}{|c|}{$15.92 \pm 7.04$} & \multicolumn{2}{|c|}{$13.77 \pm 5.97$} & \multicolumn{2}{|c|}{$13.73 \pm 3.78$} & \multicolumn{2}{|c|}{$13.31 \pm 5.49$} & \multicolumn{2}{|c|}{$6.0 \pm 2.75$} & \multicolumn{2}{|c|}{$13.66 \pm 3.73$} & \multicolumn{2}{|c|}{$13.15 \pm 5.75$} \\
\hline
\end{tabular}

Notes: homology grade of sequences 16S-23S of the middle ribosomal cluster and 23S rRNA I gene domain of Chlamydia trachomatis (NPL, PL, PL2, and PL3) and Chlamydophila pneumoniae (PN, PN2 and B) strains being investigated and with various Chlamydia types as published in GenBank NCBI, executed by BLAST program (http://blast.ncbi.nlm.nih.gov/Blast.cgi/); $\mathrm{hm}^{\hat{N}}$ - strains homology percentage; $\mathrm{d}^{* *}$ : percentage of difference in strains homology; *: difference in homology between the strains being investigated and other Chlamydia types $(M \pm m)$.

the plasmid-free strain Chlamydia trachomatis-NPL is genotype E. Strains of five serotypes were obtained from humans (K: 46.2\%, G: 23.1\%, E: 19.2\%, F: 7.7\%, J: 3.8\%); the plasmid carrying strain Chlamydia trachomatis-PL2 manifests as genotype G $[9,11,37]$.

Comparative analysis of the homology was carried out and the evolutionary difference in the known sequences 15S-23S of the middle ribosome section and domain I of the 23 rRNA gene of different types of chlamydia was estimated, with comparisons being made with the strains being studied (Tables 1 and 2). The nucleotide sequences of the sequenced fragment of the plasmid-free strain Chlamydia trachomatis-NPL have a 99\% homology and an evolutionary difference between the sequences of 0.672 to $0.690(s=$ 0.019) compared with the strains Chlamydia trachomatis
(A/Har-13, B/TW-5/OT, D/UW-3/CX), but it exhibits the greatest homology with Chlamydia trachomatis L2/434/BU $(100 \%)$. In this case the difference between the sequences is $0(s=0)$, which means that the sequences of the strains NPL and L2/434/BU are the same despite the fact that NPL has no plasmid. This confirms the previously established similarity between strains that have no plasmid and those that have it [33]. With regard to other species of chlamydia the difference in homology exhibited by NPL was $15.92 \pm$ $7.04 \%$, the difference in the sequences is 0.690 to 0.755 $(s=0.018-0.019)$. The plasmid-carrying strains Chlamydia trachomatis-PL obtained from monkeys and Chlamydia trachomatis-PL2 obtained from humans are 98\%-99\% homologous with Chlamydia trachomatis (plasmid carrying strains), with the difference in the sequences being 0.016 to 


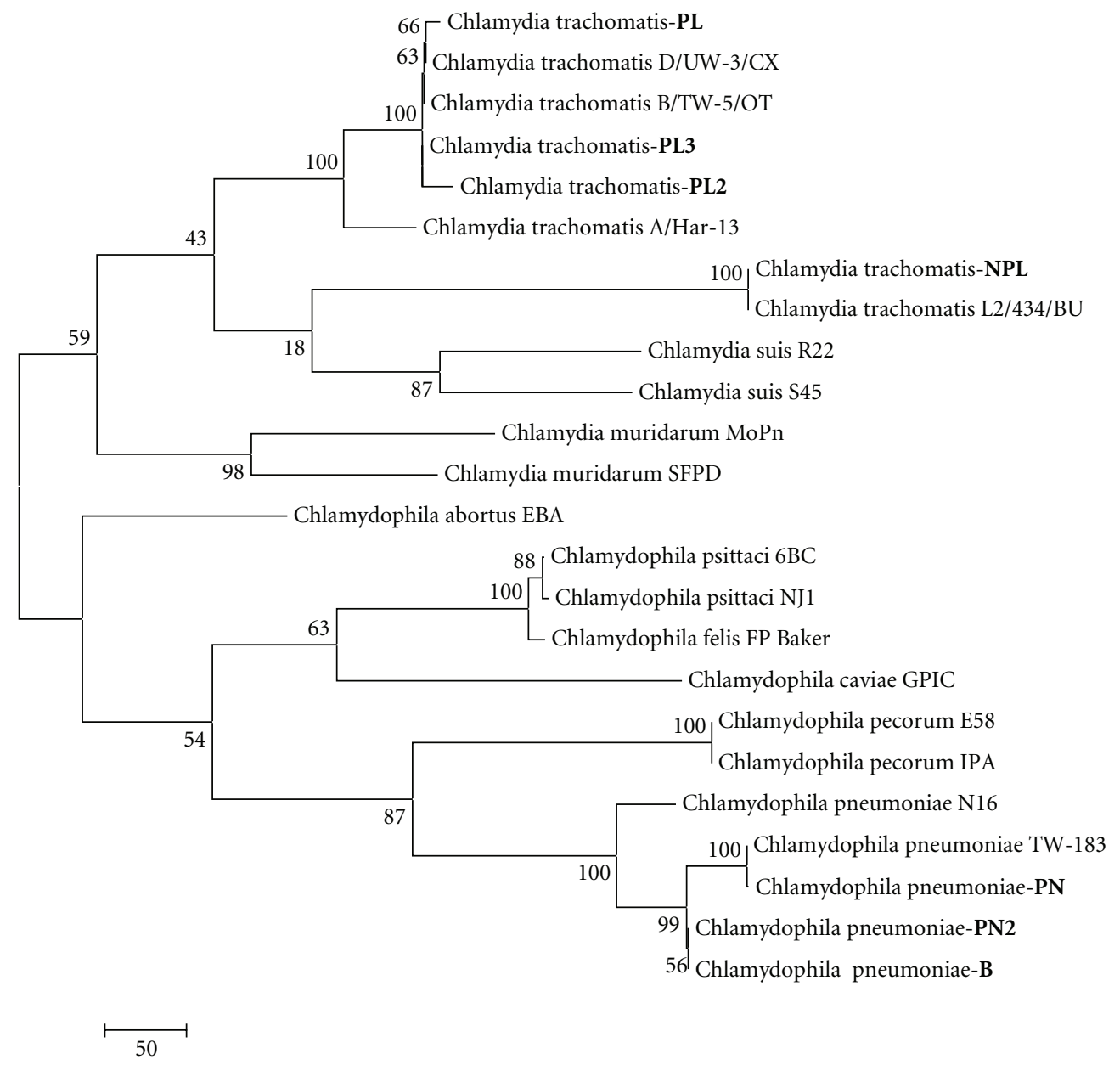

FIGURE 3: Dendrogram resulted from phylogenetic analysis of middle fragment of 16S-23S rRNA genes and 23S rRNA area of Chlamydia strains I segment by means of Maximum Parsimony method with execution of Bootstrap Test of Phylogeny (1000 repetitions). Strains being under investigation: Chlamydophila pneumoniae (PN: monkey strain, PN2: human strain, and B: reference strain) and Chlamydia trachomatis (PL: plasmid strain released from monkeys, PL2: plasmid strain released from humans, and PL3: reference strain and NPLnonplasmid strain released from monkeys).

0.697 for the strain PL $(s=0.005-0.019)$ and from 0.034 to 0.698 for PL2 ( $s=0.008-0.019$ ), with the homology differences from other types of chlamydia ranging from 13.31 $\pm 5.49 \%$ to $13.77 \pm 5.97 \%$, respectively. The nucleotide sequence of Chlamydia trachomatis-PL3 differs from that of Chlamydia trachomatis (A/Har-13, B/TW-5/OT, D/UW$3 / \mathrm{CX}, \mathrm{L} 2 / 434 / \mathrm{BU})$ by $1 \%-2 \%$, with the difference between the sequences ranging from 0.002 to 0.690 ( $s=0.002-$ 0.019). The difference in the homology of this strain and that of other species of chlamydia of both the Chlamydia and Chlamydophila genus is $13.15 \pm 5.75 \%$, with genetic distances between the sequences ranging from 0.426 to $0.691(s=0.019-0.021)$. When the evolutionary difference in the nucleotide sequences of the studied strains Chlamydia trachomatis between each other was studied, the following results were obtained: PL/PL2-0.019 $(s=0.006)$, PL/PL3: $0.052(s=0.009)$, PL/NPL: $0.697(s=0.019)$, PL2/PL3: 0.033 $(s=0.007), \mathrm{NPL} / \mathrm{PL} 3: 0.690(s=0.019)$, and NPL/PL2: 0.698 $(s=0.019)$. Consequently the plasmid-carrying monkey strain Chlamydia trachomatis-PL is the closest to the PL2 strain obtained from humans, while the plasmid-free strain NPL is closer to the reference NPL strain, but nevertheless this still could not be put in different clusters when the phylogenetic tree found in Figures 1 and 3 was constructed. Additionally, we also estimated the nucleotide sequence 16S$23 \mathrm{~S}$ of the middle ribosome section and domain I of the $23 \mathrm{~S}$ rRNA gene of the studied plasmid-carrying and plasmidfree strains Chlamydia trachomatis to get a better estimate of the aforementioned evolutionary difference in the nucleotide sequences between these strains (Figures 4-6). When comparing the monkey strains Chlamydia trachomatis-PLgenotype $\mathrm{G}$ and Chlamydia trachomatis-NPL-genotype E, a difference was identified inside a section of 483 nucleotide pairs in length (Figure 4). A similar tendency was found when Chlamydia trachomatis-PL2-genotype G (human) was compared with Chlamydia trachomatis-NPL-genotype E (monkey), where differences can be observed in a section of 197 nucleotide pairs in length (Figure 5). When Chlamydia trachomatis-PL2 (human) was compared with Chlamydia trachomatis-PL (monkey), isolated differences were found 
Chlamydia trachomatis-PL/chlamydia trachomatis-NPL

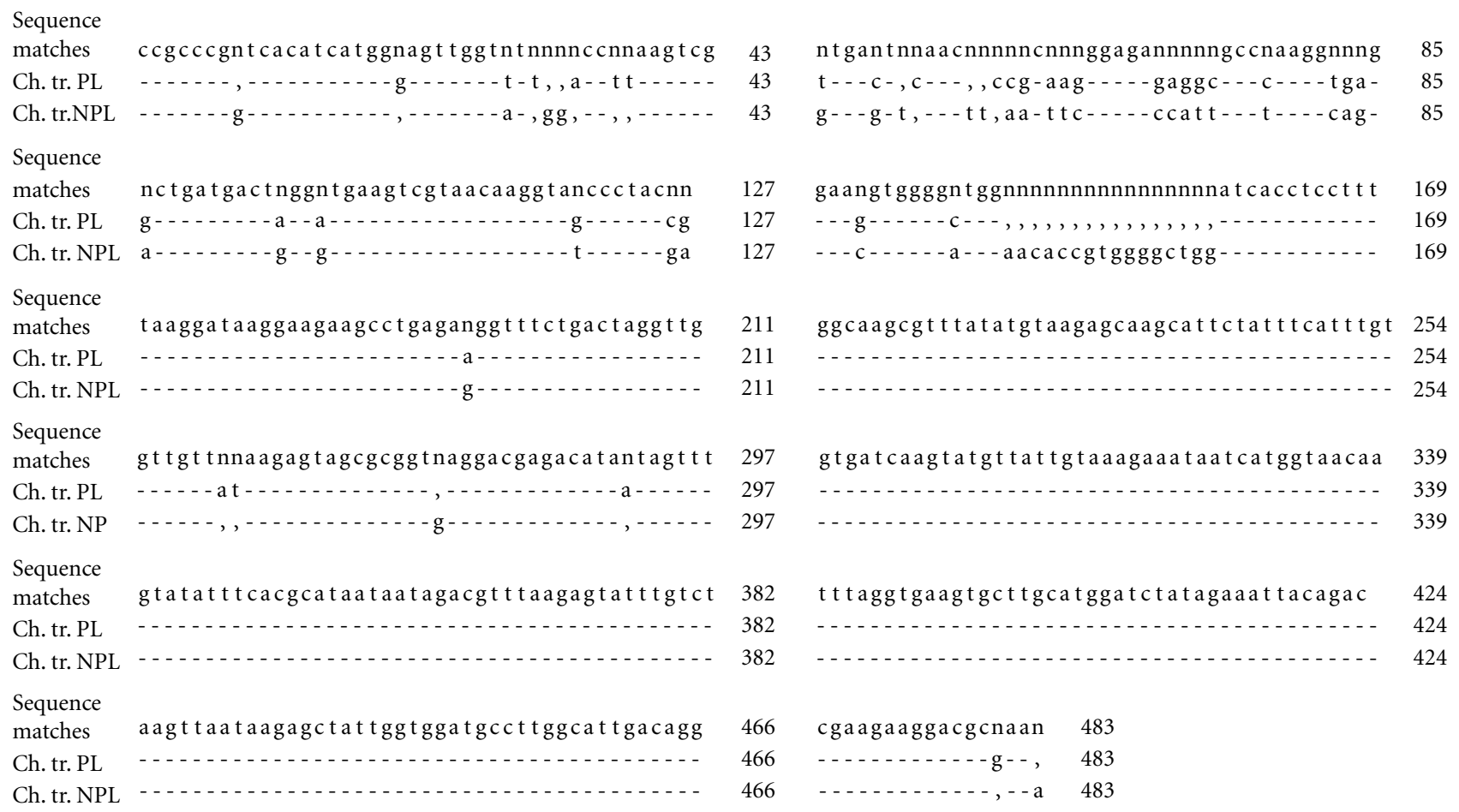

FIGURE 4: Comparative analysis of 16S-23S nucleotide sequence clusters of the middle ribosomal cluster and 23S rRNA I domain of plasmid (PL) and nonplasmid (NPL) Chlamydia trachomatis strains released from monkeys. (-) — match of nucleotides in the sequence, (n) — mismatch of nucleotides in the sequence, $($,$) _absence of nucleotides in the sequence, (43-483)—nucleotide sequence size.$

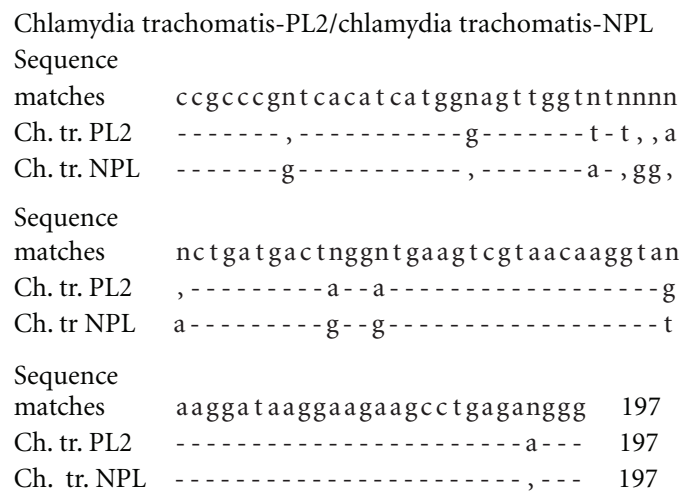

FIGURE 5: Comparative analysis of 16S-23S nucleotide sequence clusters of the middle ribosomal cluster and $23 \mathrm{~S}$ rRNA I domain of nonplasmid (NPL) Chlamydia trachomatis strains released from monkeys and plasmid (PL2) Chlamydia trachomatis strains released from humans. (-) - match of nucleotides in the sequence, (n) - mismatch of nucleotides in the sequence, (,) - absence of nucleotides in the sequence, (43-197)—nucleotide sequence size.

(within a section 462 nucleotide pairs in length); both strains are genotype G (Figure 6).

The studied strains of the Chlamydophila pneumoniae species (PN, PN2 and B) exhibit a 98\% and 99\% homology with Chlamydophila pneumoniae N16 and Chlamydophila pneumoniae TW-183, respectively. The evolutionary differences between the sequences of the strains PN2, B, TW-183, and N16 are between 0.066 and $0.128(s=0.010-0.014)$; between the monkey strain PN and TW-183 it makes 0.002 $(s=0.002)$ with a homology of $99 \%$; between PN and N16 it makes $0.190(s=0.016)$ with a homology of $98 \%$. Thus PN2, $\mathrm{B}$, and PN are close to Chlamydophila pneumoniae TW-183 in terms of homology and evolutionary differences between their nucleotide sequences. The difference in homology between PN2, B, PN, and other species is as follows: PN2: $6.0 \pm 2.75 \%$; B: $13.66 \pm 3.73 \%$; and PN: $13.73 \pm 3.78 \%$; the evolutionary difference between the nucleotide sequences varies from 0.545 to $0.719(s=0.019-0.021)$. Estimating 


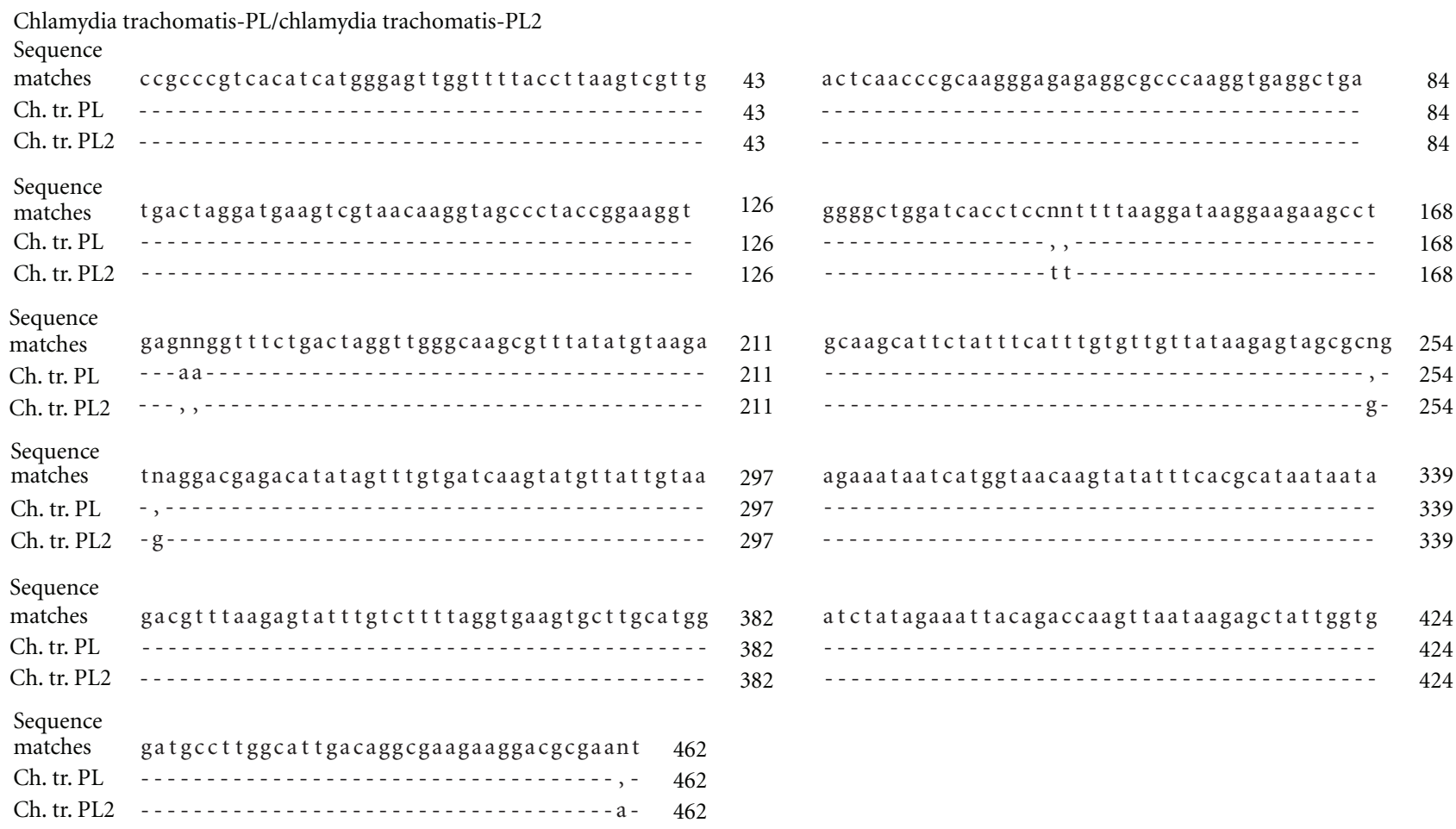

FIGURE 6: Comparative analysis of 16S-23S nucleotide sequence clusters of the middle ribosomal cluster and 23S rRNA I domain of plasmid Chlamydia trachomatis strains released from monkeys (PL strain) and from humans (PL2 strain). (-)—match of nucleotides in the sequence, (n) — mismatch of nucleotides in the sequence, (,)—absence of nucleotides in the sequence, (43-462) — nucleotide sequence size.

the evolutionary difference in the nucleotide sequences between the studied strains of Chlamydophila pneumonia (PN2: human strain, PN: monkey strain, B: reference strain), we established the following: PN2/PN: $0.067(s=0.010)$; PN2/B: $0.0(s=0)$; and PN/B: $0.067(s=0.010)$. PN2 and the reference strain $\mathrm{B}$ are the closest to each other and are at the same evolutionary level; however, the insignificance of the difference from PN means that all of the three studied strains of Chlamydophila pneumonia (human and monkey) are very similar to each other.

The homology between strain Chlamydophila pneumonia: PN, isolated from monkeys, and human strains of Chlamydophila pneumoniae amounted to $98 \%-99 \%$; plasmid-free strain Chlamydia trachomatis: NPL was completely identical to Chlamydia trachomatis L2/434/BU (homology 100\%), plasmid-carrying strain Chlamydia trachomatis: PL both with human and with known Chlamydia trachomatis strains from GenBank NCBI had 98\%-99\% homology, human Chlamydophila pneumoniae strains (PN2 and B) and Chlamydophila pneumoniae TW-183\%: 99\%; strains Chlamydia trachomatis (PL2, PL3) were homologous to Chlamydia trachomatis representatives $(98 \%-100 \%)$.

In order to examine the relationship between plasmidfree Chlamydia trachomatis-NPL and strains of Chlamydia genus phylogenetically, the dendrogram was designed ( $p$. 1 ), it shows that the strain is a representative of Chlamydia trachomatis species and is included into one cluster with Chlamydia trachomatis L2/434/BU.

The phylogenetic tree of examined plasmid-carrying Chlamydia trachomatis strains isolated from humans and monkeys (PL2 and PL), including reference strain Chlamydia trachomatis-Burkhun (PL3), with strains from Chlamydia genus is presented on the Figure 1. Strains PL, PL2, and PL3 are united into one combined cluster with Chlamydia trachomatis (B/TW-5/OT, D/UW-3/CX, A/Har-13), but into separate clusters with $\mathrm{L} 2 / 434 / \mathrm{BU}$.

The phylogenetic tree of Chlamydophila pneumoniae strains ( $\mathrm{PN}, \mathrm{PN} 2$, and $\mathrm{B}$ ) with strains from Chlamydia genus (p. 2) reflects the relationship between strains $\mathrm{PN}$ isolated from monkeys, with strain, isolated from humans (PN2), reference strain Chlamydophila pneumoniae-B and strains Chlamydophila pneumoniae TW-183, Chlamydophila pneumoniae N16. Strain PN is included into one subcluster with Chlamydophila pneumoniae TW-183, this fact suggests that the strain is a member of Chlamydophila pneumoniae species, in one cluster with Chlamydophila pneumoniaePN2 and Chlamydophila pneumoniae-B, which are in one subcluster according to evolutional discrepancy.

Phylogenetic analysis of strains Chlamydophila pneumoniae and Chlamydia trachomatis, isolated from monkeys, showed that these strains are really the members of Chlamydophila genus and Chlamydia genus according to general location in clusters on dendrograms with analogical human strains.

Considering the phylogenetic aspect of the relation between the plasmid-free strain Chlamydia trachomatisNPL with strains of the Chlamydia genus, we constructed a tree diagram (Figure 1), which indicates that this strain is a Chlamydia trachomatis and is in the same cluster as Chlamydia trachomatis L2/434/BU. 


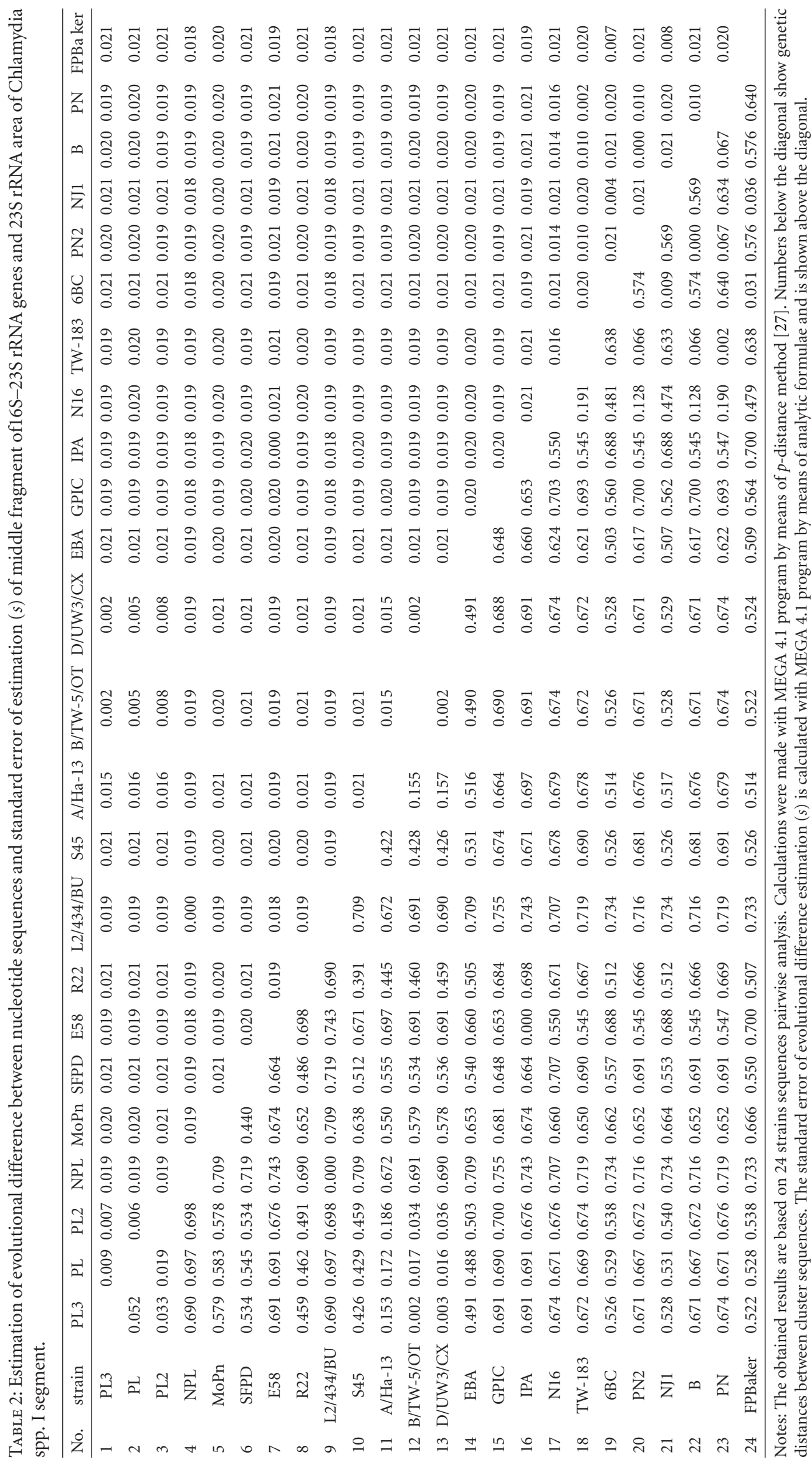


The phylogenetic tree of the studied plasmid-carrying strains of Chlamydia trachomatis, obtained from humans and monkeys (PL2 and PL), including the reference strain of Chlamydia trachomatis-Burkhan (PL3), with the strain of Chlamydia is presented in Figure 1. Strains PL, PL2, and PL3 are grouped into the same cluster with Chlamydia trachomatis (B/TW-5/OT, D/UW-3/CX, A/Har-13), but they are placed in a different cluster than L2/434/BU.

The phylogenetic tree of the strains of Chlamydophila pneumoniae (PN, PN2, and B) with strains of the Chlamydophila genus (Figure 2) reflects the relation between the PN strain obtained from monkeys with the PN2 strain obtained from humans, the reference strain of Chlamydophila pneumoniae- $\mathrm{B}$ and the strains of Chlamydophila pneumoniae TW-183, and Chlamydophila pneumoniae N16. The PN strain was place in the same subcluster with Chlamydophila pneumoniae TW-183. It is also in the same cluster with Chlamydophila pneumoniae-PN2 and Chlamydophila pneumoniae- $\mathrm{B}$, which are in the same subcluster in accordance with their small evolutionary differences.

Thus phylogenetic analysis of strains of Chlamydia trachomatis and Chlamydophila pneumoniae obtained from monkeys and humans has allowed us to establish their place in the phylogenetic tree of the Chlamydiaceae family. It has been established that there is a close evolutionary relation between the studied original species of Chlamydia and Chlamydophila and similar species about which there are records in GenBank. For the first time, it has been demonstrated that there are differences in the nucleotide sequence $16 \mathrm{~S}-23 \mathrm{~S}$ of the middle ribosome section and domain I of the $23 \mathrm{~S}$ rRNA gene of plasmid-carrying and plasmid-free strains of Chlamydia trachomatis obtained from monkeys and humans, if they have different genotypes (group B- B, Ba, D, Da, E, L1, L2, L2a; intermediary groupF, G, Ga). Malfunction of the expression of the chromosome gene incA that leads to a breakdown in the development and life cycle of chlamydia and their virulence may also be linked to possible changes in the nucleotide sequence of this gene.

\section{References}

[1] K. D. E. Everett, R. M. Bush, and A. A. Andersen, "Emended description of the order Chlamydiales, proposal of parachlamydiaceae fam. nov. and Simkaniaceae fam. nov., each containing one monotypic genus, revised taxonomy of the family Chlamydiaceae, including a new genus and five new species, and standards for the identification of organisms," International Journal of Systematic Bacteriology, vol. 49, no. 2, pp. 415-440, 1999.

[2] H. Fukushi and K. Hirai, "Proposal of Chlamydia pecorum sp. nov. for Chlamydia strains derived from ruminants," International Journal of Systematic Bacteriology, vol. 42, no. 2, pp. 306-308, 1992.

[3] J. C. Hartley, S. Kaye, S. Stevenson, J. Bennett, and G. Ridgway, "PCR detection and molecular identification of Chlamydiaceae species," Journal of Clinical Microbiology, vol. 39, no. 9, pp. 3072-3079, 2001.

[4] K. D. E. Everett and A. A. Andersen, "The ribosomal intergenic spacer and domain I of the $23 \mathrm{~S}$ rRNA gene are phylogenetic markers for Chlamydia spp," International Journal of Systematic Bacteriology, vol. 47, no. 2, pp. 461-473, 1997.

[5] J. T. Grayston, C.-C. Kuo, L. A. Campbell, and S.-P. Wang, "Chlamydia pneumoniae sp. nov. for Chlamydia sp. strain TWAR," International Journal of Systematic Bacteriology, vol. 39, no. 1, pp. 88-90, 1989.

[6] R. Amann, N. Springer, W. Schönhuber et al., "Obligate intracellular bacterial parasites of acanthamoebae related to Chlamydia spp," Applied and Environmental Microbiology, vol. 63, no. 1, pp. 115-121, 1997.

[7] R. J. Birtles, T. J. Rowbotham, C. Storey, T. J. Marrie, and D. Raoult, "Chlamydia-like obligate parasite of free-living amoebae," Lancet, vol. 349, no. 9056, pp. 925-926, 1997.

[8] S. Kahane, K. D. E. Everett, N. Kimmel, and M. G. Friedman, "Simkania negevensis strain $\mathrm{Z}(\mathrm{T})$ : growth, antigenic and genome characteristics," International Journal of Systematic Bacteriology, vol. 49, no. 2, pp. 815-820, 1999.

[9] F. Kong and G. L. Gilbert, "Postgenomic taxonomy of human ureaplasmas-a case study based on multiple gene sequences," International Journal of Systematic and Evolutionary Microbiology, vol. 54, no. 5, pp. 1815-1821, 2004.

[10] K. Liu, S. J. Knabel, and E. G. Dudley, "rhs genes are potential markers for multilocus sequence typing of Escherichia coli O157:H7 strains," Applied and Environmental Microbiology, vol. 75, no. 18, pp. 5853-5862, 2009.

[11] T. Matsumoto, M. Kawakubo, M. Shiohara et al., "Phylogeny of a novel "helicobacter heilmannii" organism from a Japanese patient with chronic gastritis based on DNA sequence analysis of 16S rRNA and urease genes," Journal of Microbiology, vol. 47, no. 2, pp. 201-207, 2009.

[12] Pudjiatmoko, H. Fukushi, Y. Ochiai, T. Yamaguchi, and K. Hirai, "Phylogenetic analysis of the genus Chlamydia based on 16S rRNA gene sequences," International Journal of Systematic Bacteriology, vol. 47, no. 2, pp. 425-431, 1997.

[13] F. R. Rurangirwa, P. M. Dilbeck, T. B. Crawford, T. C. McGuire, and T. F. McElwain, "Analysis of the 16S rRNA gene of microorganism WSU 86-1044 from an aborted bovine foetus reveals that it is a member of the order Chlamydiales: proposal of Waddliaceae fam. nov., waddlia chondrophila gen. nov., sp. nov," International Journal of Systematic Bacteriology, vol. 49, no. 2, pp. 577-581, 1999.

[14] M. Takahashi, P. Yukphan, Y. Yamada, K.-I. Suzuki, T. Sakane, and Y. Nakagawa, "Intrageneric structure of the genus Gluconobacter analyzed by the 16S rRNA gene and 16S-23S rRNA gene internal transcribed spacer sequences," Journal of General and Applied Microbiology, vol. 52, no. 3, pp. 187-193, 2006.

[15] C. A. Gaydos, L. Palmer, T. C. Quinn, S. Falkow, and J. J. Eiden, "Phylogenetic relationship of Chlamydia pneumoniae to Chlamydia psittaci and Chlamydia trachomatis as determined by analysis of $16 \mathrm{~S}$ ribosomal DNA sequences," International Journal of Systematic Bacteriology, vol. 43, no. 3, pp. 610-612, 1993.

[16] O. G. Grechishnikova, V. V. Slobodenyuk, V. A. Aleshkin, et al., "Phenotypic charac-teristics of Chlamydia strains released from humans and monkeys by means of culture technique," Immunopathology, Allergology, Infectology, no. 3, pp. 44-53, 2009.

[17] V. V. Slobodenyuk, V. A. Aleshkin, B. A. Lapin, et al., "PCR detection and identification of Chlamydia germs in humans and monkeys," Immunopathology, Allergology, Infectology, vol. 3, pp. 54-62, 2009.

[18] K. Tamura, J. Dudley, M. Nei, and S. Kumar, "MEGA4: molecular evolutionary genetics analysis (MEGA) software 
version 4.0," Molecular Biology and Evolution, vol. 24, no. 8, pp. 1596-1599, 2007.

[19] M. A. Pickett, J. S. Everson, P. J. Pead, and I. N. Clarke, "The plasmids of Chlamydia trachomatis and Chlamydophila pneumoniae (N16): accurate determination of copy number and the paradoxical effect of plasmid-curing agents," Microbiology, vol. 151, no. 3, pp. 893-903, 2005.

[20] N. S. Thomas, M. Lusher, C. C. Storey, and I. N. Clarke, "Plasmid diversity in Chlamydia," Microbiology, vol. 143, no. 6, pp. 1847-1854, 1997.

[21] K. S. Sriprakash and E. S. Macavoy, "Characterization and sequence of a plasmid from the trachoma biovar of Chlamydia trachomatis," Plasmid, vol. 18, no. 3, pp. 205-214, 1987.

[22] M. Comanducci, S. Ricci, R. Cevenini, and G. Ratti, "Diversity of the Chlamydia trachomatis common plasmid in biovars with different pathogenicity," Plasmid, vol. 23, no. 2, pp. 149154, 1990.

[23] Y. Azuma, H. Hirakawa, A. Yamashita et al., "Genome sequence of the cat pathogen, Chlamydophila felis," DNA Research, vol. 13, no. 1, pp. 15-23, 2006.

[24] T. D. Read, G. S. A. Myers, R. C. Brunham et al., "Genome sequence of Chlamydophila caviae (Chlamydia psittaci GPIC): examining the role of niche-specific genes in the evolution of the Chlamydiaceae," Nucleic Acids Research, vol. 31, no. 8, pp. 2134-2147, 2003.

[25] J. H. Carlson, S. F. Porcella, G. McClarty, and H. D. Caldwell, "Comparative genomic analysis of Chlamydia trachomatis oculotropic and genitotropic strains," Infection and Immunity, vol. 73, no. 10, pp. 6407-6418, 2005.

[26] N. R. Thomson, M. T. G. Holden, C. Carder et al., "Chlamydia trachomatis: genome sequence analysis of lymphogranuloma venereum isolates," Genome Research, vol. 18, no. 1, pp. 161$171,2008$.

[27] T. D. Read, R. C. Brunham, C. Shen et al., "Genome sequences of Chlamydia trachomatis MoPn and Chlamydia pneumoniae AR39," Nucleic Acids Research, vol. 28, no. 6, pp. 1397-1406, 2000.

[28] V. I. Yunkerov and S. G. Grigor'ev, Mathematical and Statistical Analysis of Medical Investigation Data, VMedA, SaintPetersburg, Russia, 2nd edition, 2005.

[29] Z. Li, D. Chen, Y. Zhong, S. Wang, and G. Zhong, "The Chlamydial plasmid-encoded protein pgp3 is secreted into the cytosol of Chlamydia-infected cells," Infection and Immunity, vol. 76, no. 8, pp. 3415-3428, 2008.

[30] E. M. Peterson, B. A. Markoff, J. Schachter, and L. M. de la Maza, "The 7.5-kb plasmid present in Chlamydia trachomatis is not essential for the growth of this microorganism," Plasmid, vol. 23, no. 2, pp. 144-148, 1990.

[31] A. Farencena, M. Comanducci, M. Donati, G. Ratti, and R. Cevenini, "Characterization of a new isolate of Chlamydia trachomatis which lacks the common plasmid and has properties of biovar trachoma," Infection and Immunity, vol. 65, no. 7, pp. 2965-2969, 1997.

[32] D. R. Stothard, J. A. Williams, B. van der Pol, and R. B. Jones, "Identification of a Chlamydia trachomatis serovar e urogenital isolate which lacks the cryptic plasmid," Infection and Immunity, vol. 66, no. 12, pp. 6010-6013, 1998.

[33] J. H. Carlson, W. M. Whitmire, D. D. Crane et al., "The Chlamydia trachomatis plasmid is a transcriptional regulator of chromosomal genes and a virulence factor," Infection and Immunity, vol. 76, no. 6, pp. 2273-2283, 2008.

[34] Y. Pannekoek, J. Spaargaren, A. A. J. Langerak, J. Merks, S. A. Morré, and A. van der Ende, "Interrelationship between polymorphisms of IncA, fusogenic properties of Chlamydia trachomatis strains, and clinical manifestations in patients in The Netherlands," Journal of Clinical Microbiology, vol. 43, no. 5, pp. 2441-2443, 2005.

[35] R. J. Suchland, D. D. Rockey, J. P. Bannantine, and W. E. Stamm, "Isolates of Chlamydia trachomatis that occupy nonfusogenic inclusions lack IncA, a protein localized to the inclusion membrane," Infection and Immunity, vol. 68, no. 1, pp. 360-367, 2000.

[36] R. J. Suchland, D. D. Rockey, S. K. Weeks, D. T. Alzhanov, and W. E. Stamm, "Development of secondary inclusions in cells infected by Chlamydia trachomatis," Infection and Immunity, vol. 73, no. 7, pp. 3954-3962, 2005.

[37] V. V. Slobodenyuk, A. V. Karaulov, V. A. Aleshkin, et al., "Genetic typing and analysis of antibiotic resistance of Chlamydia trachomatis strains released from humans and monkeys," Immunopathology, Allergology, Infectology, no. 4, pp. 74-81, 2009. 


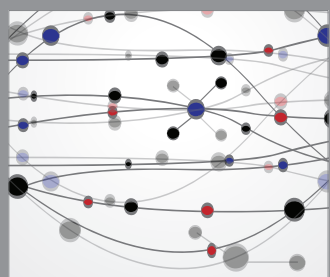

The Scientific World Journal
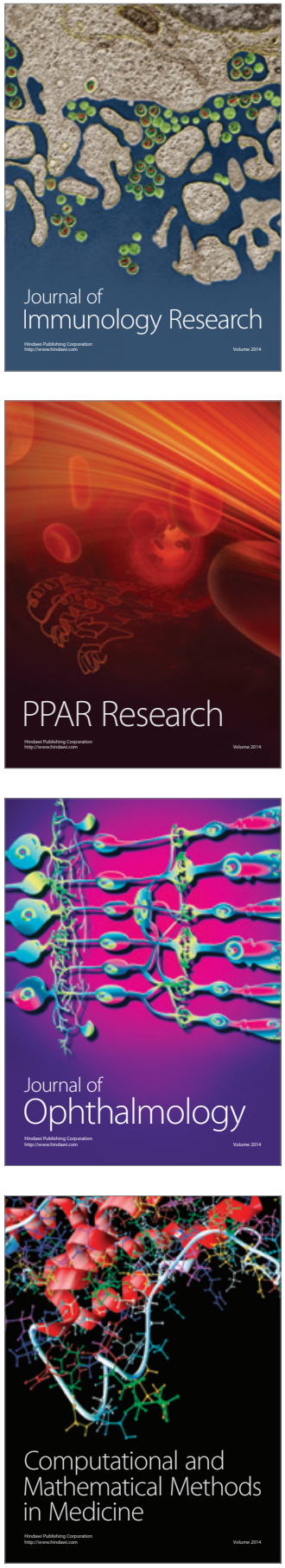

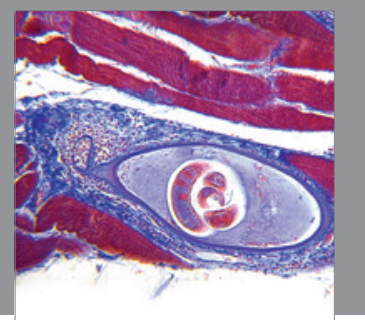

Gastroenterology

Research and Practice
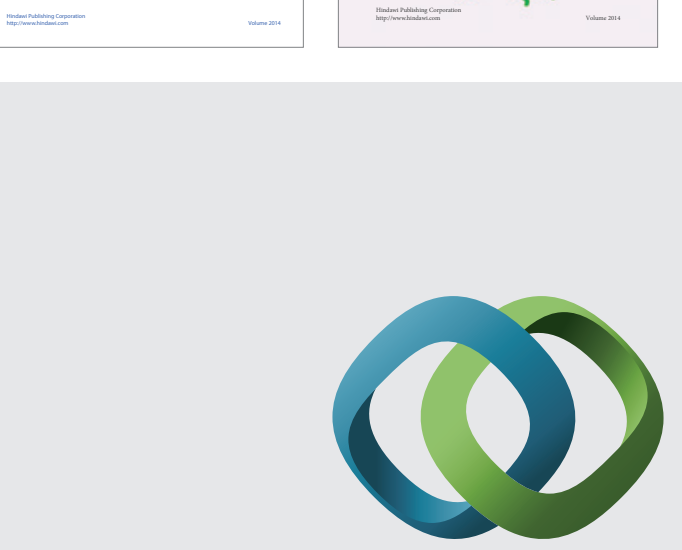

\section{Hindawi}

Submit your manuscripts at

http://www.hindawi.com


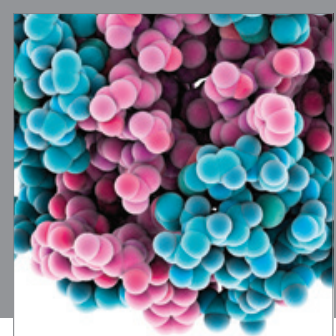

Journal of
Diabetes Research

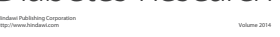

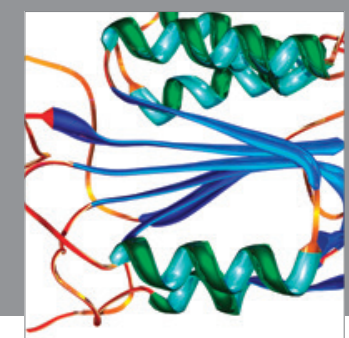

Disease Markers
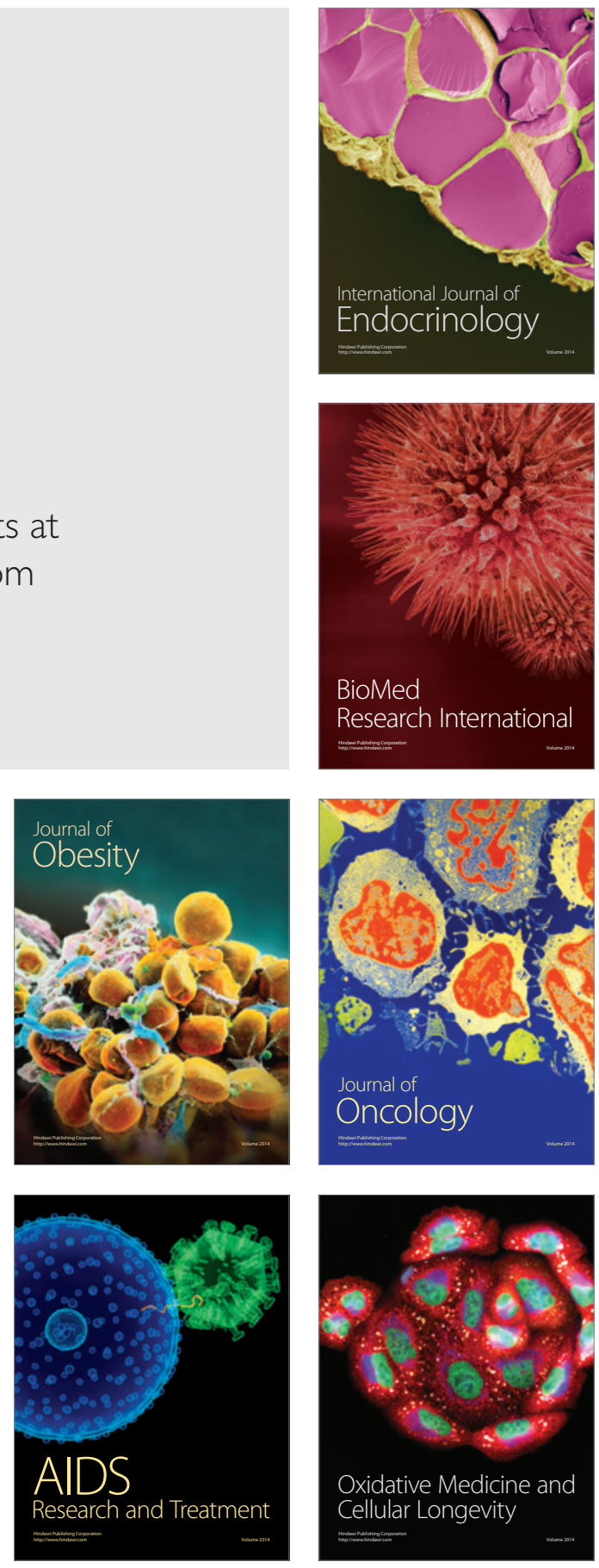